\title{
Particle Swarm Optimization for Network-based Data Classification
}

\author{
Murillo G. Carneiro, ${ }^{\mathrm{a}, *}$, Ran Cheng ${ }^{\mathrm{b}}$, Liang Zhao ${ }^{\mathrm{c}}$, Yaochu Jin ${ }^{\mathrm{d}}$ \\ ${ }^{a}$ Faculty of Computing, Federal University of Uberlândia, Uberlândia, MG, 38400-902, \\ Brazil \\ ${ }^{b}$ School of Computer Science, University of Birmingham, Birmingham, B15 2TT, UK \\ ${ }^{c}$ Department of Computing and Mathematics, University of São Paulo, Ribeirão Preto, SP, \\ 14040-901, Brazil \\ ${ }^{d}$ Department of Computer Science, University of Surrey, Guildford, GU2 7XH, UK
}

\begin{abstract}
Complex networks provide a powerful tool for data representation due to its ability to describe the interplay of topological, functional, and dynamical properties of the input data. A fundamental process in network-based (graph-based) data analysis techniques is the network construction from original data usually in vector form. Here, a natural question is: How to construct an "optimal" network regarding a given processing goal? This paper investigates structural optimization in the context of network-based data classification tasks. To be specific, we propose a bio-inspired optimization framework which is responsible for building a network from vector-based data set while optimizing a quality function driven by the classification accuracy. The classification process considers both topological and physical features of the training and test data and employing PageRank measure for classification according to the importance concept of a test instance to each class. Results on artificial and real-world problems reveal that data network generated using structural optimization provides better results in general than those generated by classical network formation methods. Moreover, this investigation suggests that other kinds of network-based machine learning and data mining tasks, such as dimensionality reduction and data clustering, can be
\end{abstract}

\footnotetext{
* Corresponding author

Email address: mgcarneiro@ufu.br (Murillo G. Carneiro)
}

Preprint submitted to Neural Networks

October 15, 2018 
also benefited by the proposed structural optimization method.

Keywords: Complex Networks, Machine Learning, Network Structural

Optimization, Data Classification, Graph Optimization, Particle Swarm.

\section{Introduction}

Complex networks refer to large scale graphs with nontrivial connection patterns. Such research subjects have triggered much interests from various fields including mathematics, physics, biology, medicine, economy, sociology, and engineering [1, 2, 3, 4, 5].

Literature presents various complex network models, such as random, smallworld, and scale-free networks [6, 7, 8]. As a common point, most complex network models have been built to satisfy certain network measures. For example, in their seminar work [7, the authors proposed a scale-free network construction method with growth mechanism and preferential attachment, where the degree distribution follows a power law. Network optimization has also been studied, an example presented in [9] is the hub-and-spoke design of the airline networks, which optimizes the efficiency by ensuring high location and, at the same, providing short journeys for passengers. Another study related to network structural optimization is presented in [10, where the authors introduce a quality function composed by two opposite measures: average degree and average geodesic distance of a given network. As result, the authors found four major models of networks: sparse exponential-like model, scale-free model, highly dense model and star model.

In the last decades, we have witnessed increasing interests in network-based or graph-based machine learning due to its ability to reveal connection pattern of data 11. One of the fundamental questions in this research topic is the construction of suitable networks from vector-based data sets. Different networks built from the same data set may lead to quite different machine learning results. A natural question here is how to build a good or even an optimal network from a given data set with restrict to a specific learning task? This is certainly 
a hard and general problem and it also possesses board interests. This paper endeavors to take a step in this direction. In summary, the main contribution of this paper is to provide a method to find out the optimal network for a given data set in the context of data classification.

Several network construction methods have been developed so far. Given the similarity matrix $S$ of the original data set, one direct approach of building a network is to establish links between pairs of nodes with weights according to $S_{i j}$. However, this approach frequently leads to the emergence of complete networks which are quite similar to the original similarity matrix representation. In addition, there are two direct and general methods:

1. $k$-nearest neighbors network $(k N N)$. In this case, each instance in the data vector is represented by a node, where node $v_{i}$ is connected to node $v_{j}$ if and only if $v_{j}$ is among the $k$ most similar elements to $v_{i}$. In this way, the method results in a directed network. There are also some variations of $k$ NN. For example, the Symmetric $k \mathrm{NN}(\mathrm{S} k \mathrm{NN})$ [12], which generates a symmetric adjacency matrix $A^{\prime}$ from the original adjacent matrix $A$ obtained by $k \mathrm{NN}$ applying $A_{i j}^{\prime}=\max \left(A_{i j}, A_{i j}^{T}\right)$. Another one is the Mutual $k \mathrm{NN}(\mathrm{M} k \mathrm{NN})\left[12\right.$ where the symmetric adjacency matrix $A^{\prime}$ is obtained by applying $A_{i j}^{\prime}=\min \left(A_{i j}, A_{i j}^{T}\right)$. One problem with this kind of methods is that the best $k$ value is previous unknown; another problem is that rarely a unique value $k$ can fit well to different details of the network.

2. $\epsilon$-radius network. This method constructs an undirected network where the edge set consists of pairs $\left(v_{i}, v_{j}\right)$ such that $S_{i j}>=\epsilon$. The degree of each node can vary a lot according to the distribution of the original data. However, again we have problem to determine a proper value for $\epsilon$.

The $k$-nearest neighbors and the $\epsilon$-radius techniques treat data items in a uniform manner in dense and sparse regions. Several network formation techniques have been proposed in literature by employing adaptive information. 55 Some examples are:

- Network formation using combinations of the $k$-nearest neighbors and $\epsilon$ - 
radius techniques [13]. Here, the network formation technique activates the $k$-nearest neighbors criteria at sparse regions and employs the $\epsilon$-radius criteria in dense regions.

- $b$-matching network [14. As opposed to the $k \mathrm{NN}$ network, the $b$-matching network ensures that each node in the network has the same number of edges and therefore produces a regular network.

- Linear neighborhood network [15. The idea is to approximate the entire network by a series of overlapped linear neighborhood patches, and the edge weights in each patch are determined by a standard quadratic programming procedure.

- Network formation using clustering heuristics [16. This method uses data clustering heuristics to perform the network formation process. Specifically, it employs the single-link method, which is a clustering heuristic capable of constructing connected and sparse networks, while maintaining the cluster structure of the original data set.

- AdaRadius. In [17, the authors proposed an adaptive network construction method based on minimum spanning tree and $\epsilon$-radius, henceforth, called AdaRadius. Firstly, the network skeleton is obtained through the minimum spanning tree of the original data set. Then, the $\epsilon_{i}$ value of each node is estimated as the length of its longest path in the minimum spanning tree. Finally, the network is constructed using $\epsilon$-radius method.

- $k$-Associated Optimal Graph ( $k$ AOG) method [18. Most task-driven network formation methods are developed for unsupervised learning and semi-supervised learning, while $k$ AOG method is designed for supervised learning (data classification) tasks. As $k \mathrm{NN}$, the $k \mathrm{AOG}$ method creates a directed link from vertex $v_{i}$ to $v_{j}$ according to $k \mathrm{NN}$ and a purity measure, which expresses the level of mixture of a component in relation to other components of distinct classes by using the label information of the training set. Basically, $k$ AOG method merges the subsequent $k$ NN networks 
by increasing $k$, while increasing or keeping the purity of the network encountered so far, until the optimal network measured by purity is reached.

Basically, simple network construction methods, such as $k \mathrm{NN}$ and $\epsilon$-radius, considers only local data relationships and they are generally-purposed ones, i.e.,

90 tasks. On the other hand, sophisticated methods consider both local and global relationship of the input data, but they are usually used for a specific purpose. For example, network formation using clustering heuristics constructs networks aiming at data clustering tasks; $k$ AOG method is designed for data classificamethod. Here, we are particularly interested in investigating network structural optimization for the data classification task due to the following reasons:

- Network-based Data Classification. Complex networks have been widely applied to unsupervised and semi-supervised learning tasks, such as data clustering, transductive learning and dimensionality reduction 19, 20, 21, 22, 23, 24, 25, 26, 27. As there are many unlabeled data instances in unsupervised and semi-supervised learning, there is large space for labelling or other information propagation process. On the other hand, in the supervised learning setting, there is only one or a very small number of unlabeled instance, thus leaving no space for label propagation. Consequently, the literature contains few investigations about network-based data classification [18, 13, 28, 29, 30, 31, 32.

- Topological Features. Let us consider Fig. 1|(a), which shows a simple data set with very clear patterns of two classes: the circle data items (blue colored) and the square data items (red colored). The triangle data items (black colored), which represent the test instances to be classified, are clearly the continuation of the Grid pattern formed by the elements of the circle class. However, traditional classification techniques usually have trouble in detecting the test items as belonging to that class, as they perform classification only based on the physical features of the data (e.g. 


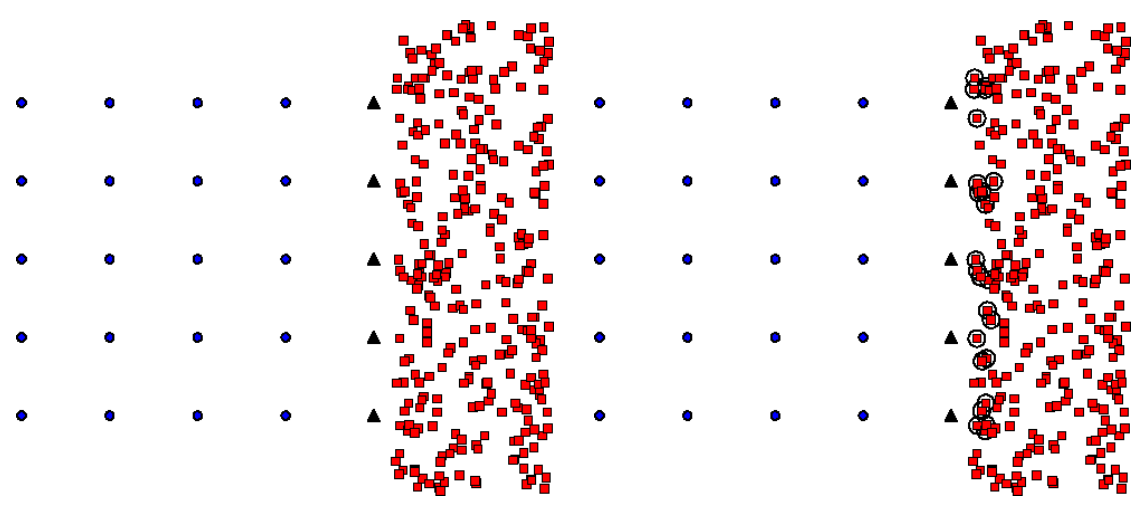

(a) Two-class data set

(b) $k$-NN (Nearest Neighbors)

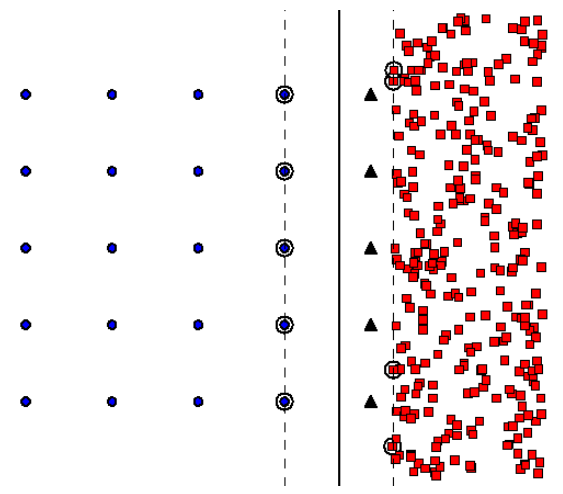

(c) SVM (Support Vectors)

Figure 1: Analysis of the classification process of traditional techniques. (a) A toy two class data set with clear patterns denoted by Blue/circle and Red/square data items, and Black/triangle data items which needs to be classified; (b) $k$-nearest neighbors classifies a test instance by verifying the label of its $k$ nearest neighbors (circulated in the figure); (c) Support Vector Machine takes into account the support vectors (circulated in the figure) to approximate each class with a convex hull and to find the best separating hyperplane between the classes, which is used as a decision boundary to classify test instances. Traditional classification techniques usually label the triangle data items as belonging to the square class because they are unable to identify the semantic pattern formation of the data, i.e., the clearly continuation of the Grid pattern formed by the elements of the circle class. 
similarity, distribution or distance). Instead, such techniques will classify the test instances to the square class, as showed in Figs. 1](b) and 1][(c) This is because many square class elements physically stay near the test instances. By analyzing the decision process behind $k$-nearest neighbors $(k-\mathrm{NN})$ and Support Vector Machine (SVM) in the figures, one can see that traditional techniques really fail to identify semantic patterns formed by the data. For example, $k$-NN classifies a test instance by verifying the label of its $k$ nearest neighbors (circulated in Fig. 1](b)), and SVM takes into account the support vectors (circulated in Fig. 11(c) to approximate each class with a convex hull and to find the best separating hyperplane between the classes. By contrast, by examining the pattern formation among the data instances, complex networks are expected to capture spatial, functional and topological structures of the data.

By the reasons presented above, this work proposes a framework to perform structural optimization for network-based data classification. In contrast in setting a specific threshold value of connections by all nodes (or groups of nodes) in related works, we propose that connections between nodes are iteratively updated by a bio-inspired optimization framework. The framework is expected to build up the network while conducting the optimization of a quality function. ${ }_{335}$ In summary, the list of main contributions presented in this article includes:

- The design of a bio-inspired framework able to generate optimized networks constructed from vector-based data sets. Despite any populationbased algorithm can be adopted in the framework, the social learning particle swarm optimization (SL-PSO) 33] has been adopted in this article due to its robust performance against several state-of-the-art PSO variants on dozens of high dimensional benchmark functions.

- A range of machine learning tasks can be learned by the proposed framework as the quality function is task-driven, i.e., the framework is able to perform structural optimization for data classification, dimensionality 
reduction and outlier detection, just to name a few. In this article, we investigate the optimization of networks in the data classification task. The quality function is driven by the importance-based classification [32, which exploits topological and physical features of the networked data and employs PageRank concepts to classify a test instance into the network component (class) having more importance.

- The design of two mapping heuristics able to provide efficient network representation and manipulation for the bio-inspired framework. The mapping heuristics generates a particular adjacency list which defines for each vertex its candidate vertices to link based on specific criteria.

- Experiments against widely used classification methods, such as the stateof-the-art Convolutional Neural Networks (CNN) and SVM, show that the salient features of the network-based classification method are highlighted by the optimized network.

- Results on artificial and real-world data sets reveal that the network provided by the framework provides statistically better results than those generated by other network formation methods, especially in higher complexity of class configuration (such as the mixture among different classes).

The remainder of the paper is organized as follows. A relevant background is provided in Sect. 2. The framework proposed for structural optimization is detailed in Sect. 3. Computer simulations and discussions about the results obtained on artificial and real-world data sets are presented in Sect. 4. Section 5 provides a general discussion about the main contributions of this work.

\section{Background}

Here we present a brief description about the techniques related to this work. Firstly, Sub-Sect. 2.1 formalizes network-based data classification. Then, SubSect. 2.2 introduces the importance-based classification tecnique and Sub-Sect. 2.3 gives a quick overview about the SL-PSO algorithm. 


\subsection{Network-based Data Classification}

In the data classification problem considered here, the algorithms receive as

175

\subsection{Importance-based classification}

The importance-based classification is a recently proposed technique based on complex network measures [32]. In contrast to traditional techniques that perform classification only based on the physical features of the data, e.g. similarity, distance, or distribution, the importance-based classification considers both physical and topological features to classify a test instance into a network component (class). The importance here is characterized by PageRank measure. 
Formally, the importance $I$ of a test instance $y$ with respect to the class $\mathcal{C} \in \mathcal{L}$ is given by:

$$
I_{y}^{(\mathcal{C})}=\sum_{v_{j} \in \Lambda_{y}^{\mathcal{C}}} I_{j}
$$

where $v_{j} \in X_{\text {train }}$ denotes a labeled node, $\Lambda_{y}^{\mathcal{C}}$ is the set of nodes pertaining to the class $\mathcal{C}$ where $y$ is temporarily connected, and $I_{j}$ means the importance of node $v_{j}$. Each node $v_{j}$ is quantified in terms of importance $\left(I_{j}\right)$ by iterating the following system:

$$
I_{j}^{(t+1)}=\sum_{i \rightarrow j} \beta \cdot \frac{I_{i}^{(t)}}{d_{i}}+(1-\beta) \frac{1}{n},
$$

which corresponds to the PageRank formulation [34].

The set of temporary links to $y$, denoted as $\Lambda_{y}^{\mathcal{C}}$ in Eq. 1, is obtained from the following formulation:

$$
\Lambda_{y}^{\mathcal{C}} \cup\left\{v_{j} \mid \mathcal{F}(y, j) \geq 0 \text { and } l_{j} \in \mathcal{C}\right\}
$$

where $\mathcal{F}_{y, j}$ is a function that verifies if a link between $y$ and $j$ increases or decreases the efficiency of the component containing the node $j$. If it increases the efficiency, then vertex $j$ is added to the $\Lambda_{y}^{\mathcal{C}}$ set. The mathematical formulation of $\mathcal{F}_{y, j}$ is given by:

$$
\mathcal{F}_{y, j}=\underset{v_{j} \in \alpha}{\mathcal{E}^{\alpha}} \cdot \gamma-D_{y, j}
$$

where $D_{y, j}$ refers to the distance between nodes $y$ and $j, \mathcal{E}^{\alpha}$ is the efficiency of the component $\alpha$, and $\gamma$ is a parameter which fits the component efficiency values obtained from the network formation method. Note that when $\gamma=1.0$, the technique takes the efficiency patterns as obtained by the network formation methods. More specifically, the efficiency of a component $\mathcal{E}^{\alpha}$ can be defined as the average value of the local efficiency $\xi$ of the nodes that belong to $\alpha$, i.e.,

$$
\mathcal{E}^{\alpha}=\frac{1}{N^{\alpha}} \sum_{i \in \alpha} \xi_{i}^{(\alpha)}, \quad \xi_{i}^{(\alpha)}=\frac{1}{N_{i}} \sum_{i \rightarrow j} D_{i, j}
$$

where $N^{\alpha}$ denotes the number of nodes in the component $\alpha, N_{i}$ denotes the number of links from $i$ and $D_{i, j}$ is the Euclidean distance between nodes $i$ and $200 \quad j$. 
The illustrative example in Fig. 1](a) is a toy data set with two classes presenting strong patterns. It serves as a tool for better motivating the study of network-based techniques as it emphasizes particular situations in which traditional techniques have trouble in correctly classifying the unlabeled items.

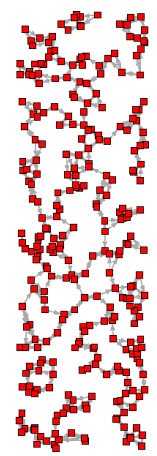

(a) Network formed from Fig. 11 a)

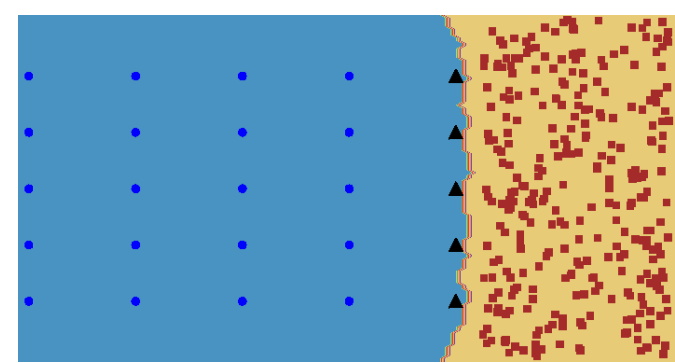

(b) Importance-based Classification

Figure 2: Analysis of the classification provided by the importance-based data classification. (a) network formed from the data set is presented by Fig. 11(a) (b) by exploiting topological, functional and spatial properties represented through the network connections, the importance-based technique is able to detect semantic relationship among the data and it consequently classifies the triangle data items as belonging to Blue/circle class.

\subsection{Social learning particle swarm optimization}

The social learning particle swarm optimization algorithm (SL-PSO) is a recently proposed swarm intelligence paradigm for large scale optimization [33. In contrast to traditional particle swarm optimization (PSO) algorithms [35, 36, 
SL-PSO does not memorize the historical best positions - neither the global best position gebst nor the personal best position pbest. Instead, in SL-PSO, the swarm is sorted according the fitness values of the particles, and as a consequence, each particle is made to learn from any better particles in the current swarm as follows:

$$
s_{i, j}(t+1)=\left\{\begin{array}{l}
s_{i, j}(t)+\operatorname{vel}_{i, j}(t+1), \text { if } p_{i}(t) \leq \operatorname{Prob}_{i}^{L} \\
s_{i, j}(t), \text { otherwise }
\end{array}\right.
$$

where $t$ is the generation counter, $s_{i, j}(t)$ is the $j$-th $(j \in\{1,2,3, \ldots, D\})$ dimension in the position vector of particle $i(i \in\{1,2,3, \ldots, m\})$, with $m$ and $D$ denoting the swarm size and number of decision variables, respectively; $s_{i, j}(t)$ is updated according to velocity vel $_{i, j}(t+1)$ based on a learning probability Prob ${ }_{i}^{L}$ for each particle $i$ in the sorted swarm; $\epsilon$ is a parameter known as the social influence factor; $F_{1}(t), F_{2}(t)$ and $F_{3}(t)$ are three coefficients randomly generated within $[0,1]$. In detail, $\operatorname{vel}_{i, j}(t+1)$ is generated as follows:

$$
\begin{aligned}
\operatorname{vel}_{i, j}(t+1) & =F_{1}(t) \cdot \operatorname{vel}_{i, j}(t) \\
& +F_{2}(t) \cdot I_{i, j}(t)+F_{3}(t) \cdot \varepsilon \cdot S_{i, j}(t),
\end{aligned}
$$

where $\operatorname{vel}_{i, j}(t+1)$ consists of three components: the inertia component $F_{1}(t)$. vel $_{i, j}(t)$, the imitation component $I_{i, j}(t)$ and the social influence component $S_{i, j}(t)$. The inertia component is similar to that in traditional PSO, while the the imitation component and the social influence component are inspired from the social learning theories as:

$$
\left\{\begin{array}{l}
I_{i, j}(t)=s_{k, j}(t)-s_{i, j}(t) \\
S_{i, j}(t)=\bar{s}_{j}(t)-s_{i, j}(t)
\end{array}\right.
$$

where $s_{k, j}(t)$ is the $j$-th dimension in the position vector of particle $k$, known as a demonstrator, which has a better fitness value than particle $i ; \bar{s}_{j}(t)=\frac{\sum_{i=1}^{m} s_{i}^{j}}{m}$ is the mean position of the swarm in generation $t$. 


\section{Bio-inspired optimization framework}

In this section, the bio-inspired optimization framework is described in details. Sub-Sects. 3.1, 3.2 and 3.3 present, respectively, an overview about our framework; a complete description about the training and test phases; and a discussion about the computational complexity of the technique.

\subsection{Overview}

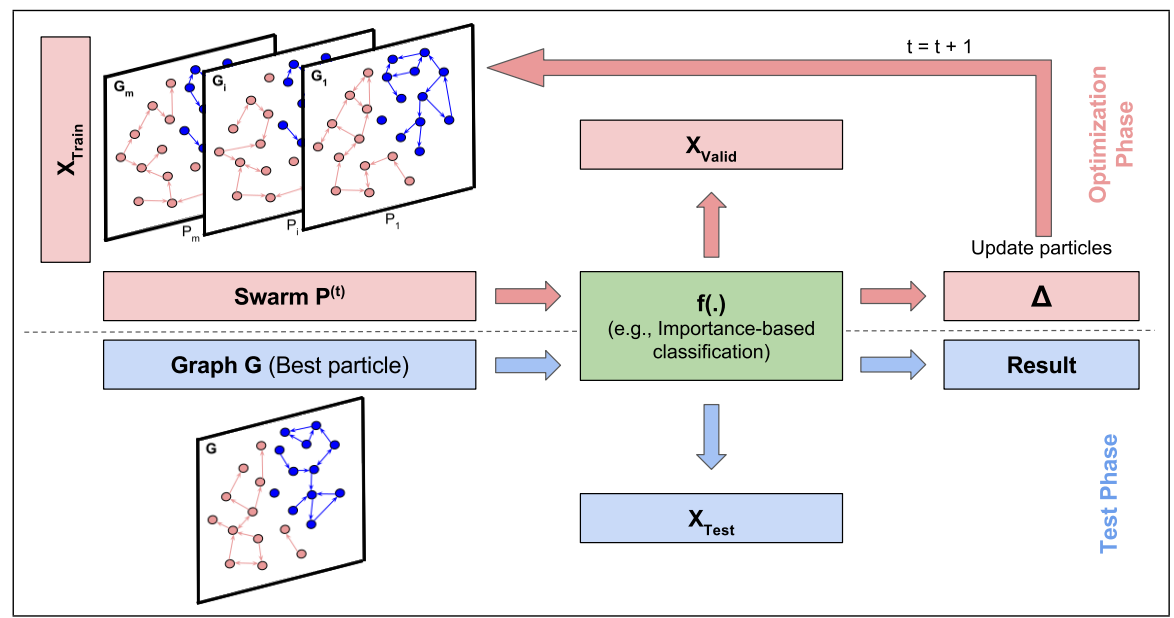

Figure 3: General framework for structural optimization in network-based learning. The illustration emphasizes the two phases of the framework: optimization phase (also called training phase) and test phase.

The optimization framework proposed in this paper is divided in two phases. The first one is the optimization phase (or training). In this phase, SL-PSO algorithm is used to construct the network from the training data $X_{\text {train }}$ optimizing a given quality function $f$ under a given validation data set $X_{\text {valid }}$. The second phase is the test phase, where the best solution in the training phase is equipped by $f$ to classify each new instance $y \in X_{\text {test }}$. Here, the function $f$ gives the predictive accuracy by using a simplified version of the network-based classification technique proposed in 32. The main steps of the general framework for structural optimization are illustrated by Fig. 3. Initially, SL-PSO creates 
a population of particles, where each particle $\mathcal{P}_{i} \in P$ represents a network $\mathcal{G}_{i}$. Then, at each generation $t$, the particles are evaluated and updated $(\Delta)$ according to a quality function $f$. At the end, SL-PSO returns the particle with the best fitness value, which contains the network to be adopted in the test phase 235 in order to classify every unknown instance in $X_{\text {test }}$.

\subsection{Framework description}

The optimization phase in the proposed framework mainly consists of the following three modules.

\subsubsection{Optimization framework}

The optimization framework is driven by the SL-PSO algorithm, which is a recently proposed bio-inspired algorithm for large scale optimization. The main steps of the algorithm are presented in Alg. 1. At each generation, SL-PSO evaluates and updates the particles according to the better particles in such a way that each particle learns from any other better ones. The algorithm stops when the predefined maximum number of generations is reached. More details of SL-PSO can be referred to Section 2.3 .

\subsubsection{Network representation and mapping heuristics}

In order to be manipulated by the bio-inspired and network-based techniques, we designed an efficient computational representation for the networks. Given a population of $m$ particles $P=\left\{\mathcal{P}_{1}, \ldots, \mathcal{P}_{m}\right\}$, each particle $\mathcal{P}_{k} \in P$ is represented by:

$$
\mathcal{P}_{k}=\left\{v_{1}, \ldots, v_{n}\right\}
$$

where $n$ denotes the number of instances (or nodes). Each node $v_{i} \in \mathcal{P}_{k}$ denotes a labeled instance $x_{i} \in X_{\text {train }}$ and its links are represented by:

$$
v_{i}=\left\{e_{i 1}^{\prime}, \ldots, e_{i q}^{\prime}\right\}
$$

with $q$ denoting the maximum number of links and $e_{i j}^{\prime}$ the probability of a link

between two nodes. The values assumed by $e_{i j}^{\prime}$ are continuous and vary between 


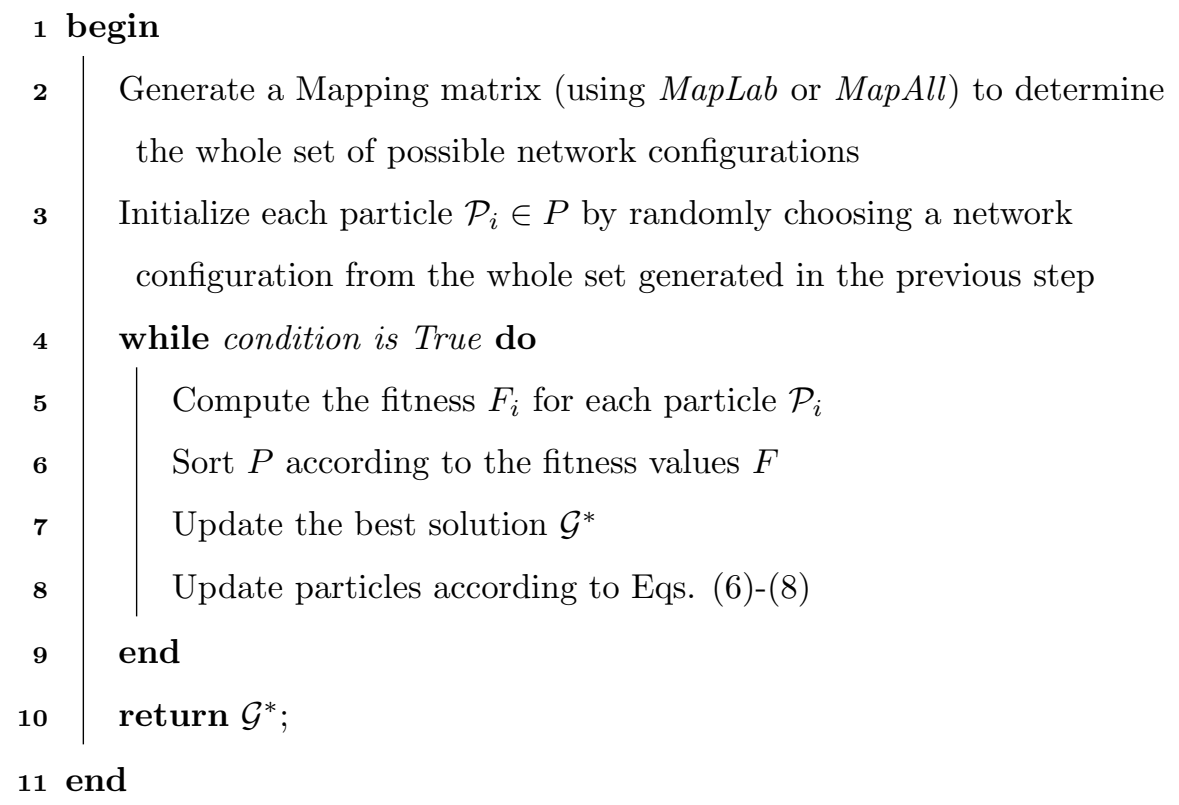

Algorithm 1: SL-PSO algorithm

$[0,1]$ in order to be manipulated by SL-PSO. On the other hand, the topological properties of the network are captured through connections, i.e. binary values, denoted as $e_{i j}$, which are obtained as:

$$
e_{i j}= \begin{cases}1, & \text { if } e_{i j}^{\prime} \geq 0.5 \\ 0, & \text { otherwise }\end{cases}
$$

To calculate the quality function of each particle $\mathcal{P}_{k}$, we convert $\mathcal{P}_{k}$ into a network $\mathcal{G}_{k}=\left\{\mathcal{V}_{k}, \mathcal{E}_{k}\right\}$, where $\mathcal{V}_{k}=\{1, \ldots, n\}$ denotes the vertices associated to each data item and $\mathcal{E}_{k}$ the edges between such vertices, which is provided by our framework. Given that a network has $n$ nodes, the complexity of the search space is $\mathcal{O}\left(n^{2}\right)$ since it is given by the total number of possible edges. For the optimization framework, however, since $n$ can be as large as hundreds or even thousands, a search complexity of $\mathcal{O}\left(n^{2}\right)$ is unfeasibly expensive. To address 255 this issue, we designed mapping heuristics that create a sub-dimensional space of size $q$ based on the features of the given data set $X_{\text {train }}$, which reduces the search complexity from $\mathcal{O}\left(n^{2}\right)$ to $\mathcal{O}(n)(\mathcal{O}(n \cdot q), q \ll n)$, where $q$ is the 
maximum number of possible edges. Two mapping heuristics are proposed in this manuscript: MapLab and MapAll. The former aims to emphasize the structure of classes through connections, which can be a very useful feature on data sets characterized by very strong patterns. The latter considers the mixture among classes through the network structure, which is a promising feature when dealing with data sets characterized by complex patterns and noise. The two heuristics are detailed in the following.

- The MapLab mapping heuristic comprises these steps:

a) Compute the similarity between each pair of data items;

b) For each node $v_{i}$, select its $q$ most similar nodes (measured by Euclidean distance) that belong to the class $l_{i}$;

c) Create $M a p_{n \times q}$ matrix, where:

$$
\operatorname{Map}_{i z}=v_{z} .
$$

- The MapAll mapping heuristic comprises these steps:

a) Compute the similarity between each pair of data items;

b) For each vertex $v_{i}$, select its $q$ most similar nodes (measured by Euclidean distance);

c) Create $M a p_{n \times q}$ matrix, where:

$$
\operatorname{Map}_{i z}= \begin{cases}v_{z}, & \text { if } l_{i}=l_{z}, \\ \emptyset, & \text { otherwise }\end{cases}
$$

Despite that each mapping heuristic mantains the possible connections from each vertex, the existence of every connection is indicated by the probability of links $e^{\prime}$ which is manipulated in each particle of the optimization framework. Fig. 4 presents an example illustrating the application of the mapping heuristics in the conversion of the particles to networks. Let us consider a given node $v_{j}$ as example. In the figure, step (i) shows the calculation of the possible edges from $j$, which also emphasizes the particular characteristics of MapLab and MapAll: 
MapLab considers only vertices that belong to the same class of $j$ in order to define the set of possible edges (Fig. 4(a), and MapAll considers all vertices a priori and then excludes those that do not belong to the same class of $j$ (e.g. vertex in position $q_{4}$ in Fig. 4(b)); step (ii) presents the candidate solution in terms of a probability vector, which is manipulated by SL-PSO; step (iii) shows the transformation of the probabilities in connections; and step (iv) presents the resulting edges of vertex $j$.

\subsubsection{Quality function}

In the optimization phase, each particle $\mathcal{G}_{i}$ is evaluated by a quality function $f$ under a validation data set. In the test phase, the best particle $\mathcal{G}^{*}$ is equipped by $f$ to perform the classification of all test instances one by one. With respect to $f$, we have used a simpler version of the importance-based classification proposed in 32 which takes the efficiency patterns as obtained by the network formation methods $(\gamma=1)$. Basically, it is a network-based classification technique which exploits topological and physical features of the data and employs PageRank formulation to classify a test instance into the network component where it is more important. More details about the importance-based classification can be referred to Sub-Sect. 2.2 .

\subsection{Complexity analysis}

This subsection provides an analysis about the time complexity of the optimization framework. For simplicity, the computational analysis is performed by each module presented in Sub-Sect. 3.2 .

- Network optimization: SL-PSO is used to conduct the optimization process in the proposed framework. The time complexity of the bio-inspired algorithm is about $\mathcal{O}(m d)$ by considering $d>m$, where $m$ and $d$ denote the number of particles and dimensions, respectively. Given that $d=q n$, where $n$ is the number of instances in $X_{\text {train }}$ and $q$ is the maximum number of possible edges, with $q \ll n$, the time complexity of SL-PSO in the proposed framework lies on $\mathcal{O}(m n)$. 


\section{MapLab}

1

$\cdots$

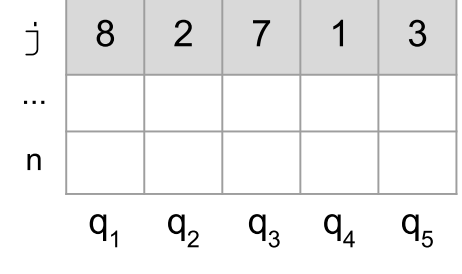

(i)

\begin{tabular}{|c|c|c|c|c|c|}
\hline (I & $q_{1}$ & $q_{2}$ & $q_{3}$ & $q_{4}$ & $q_{5}$ \\
\hline & 0.23 & 0.65 & 0.83 & 0.51 & 0.39 \\
\hline
\end{tabular}

(iii)

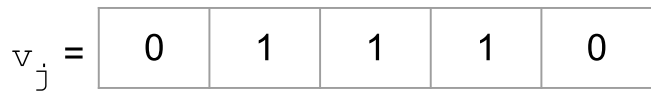

(iv)

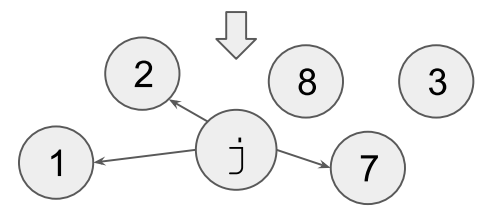

(a) MapLab

\section{MapAII}

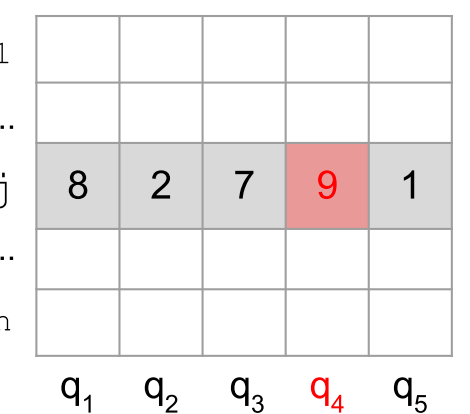

(i)

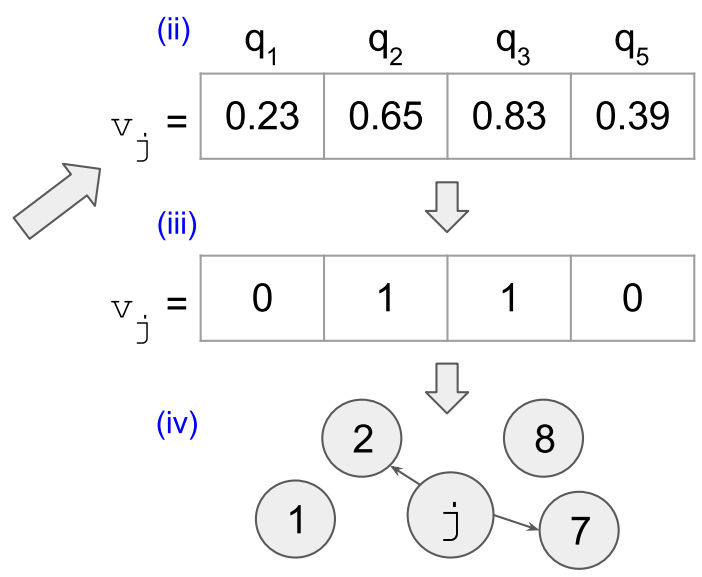

(b) MapAll

Figure 4: Illustrative example of the conversion from vector-based probability array (manipulated by SL-PSO) to network (which the structural properties are exploited) using both mapping heuristics proposed in this manuscript: (a) MapLab considers only nearest neighbors instances of the same label to create the Map matrix; (b) MapAll takes into account instances of all classes to create the Map matrix. 
- Mapping matrix: The time complexity related to MapLab and MapAll mapping heuristics lies on $\mathcal{O}\left(n^{2}\right)$ as the euclidean distance is computed between each pair of instances before the nearest neighbor search. However, this time complexity can be largely reduced by adopting any improve-

tation time can be also reduced by performing the quality function evaluations via distributed computing.

\section{Experimental results}

This section provides computer simulations in order to evaluate the proposed 335 framework. It is divided in three subsections: SubSect. 4.1 presents details about the simulations; SubSect. 4.2 provides results on artificial data sets; and SubSect. 4.3 discusses a range of experimental results performed on real-world data sets. 


\subsection{Experimental setup}

340

To be specific, we describe the parameters of our framework, the categories of techniques under comparison, the parameter selection and the statistical tests adopted.

\subsubsection{Simulations}




\subsubsection{Comparison with other network construction methods and techniques}

In addition to the bio-inspired framework proposed here, other three categories of techniques are also considered in the experiments: directed graphs,

undirected graphs and classifiers; more details about such techniques and their parameters are presented from SubSect. 4.3.2 to 4.3.4 respectively. Note that every network constructed uses the same network-based classification technique as BIO.

\subsubsection{Parameter selection}

As each data set is partitioned into three sets, training set, validation set and test set, the parameters of the techniques are selected by evaluating the predictive performance over the validation set in each execution. To be specific, we run a grid search method in each execution considering the training data and then we select the parameter which provides the best result on the validation set. Finally, the selected learning model is applied over the test set and the performance of the technique is then calculated. The process repeats until the number of executions (ten) is reached. Note that for the stochastic techniques, each execution is run for 5 times and the best average predictive performance in the validation set is considered in order to choose the parameter.

4.1.5. Statistical test

In order to analyze statistically the results obtained in the computer simulations, a statistical test that compares two methods over multiple data sets has been adopted, i.e., the Wilcoxon Signed Ranks test. The test is a non-parametric alternative to the paired t-test when its assumptions, such as normal distribution, can not be assured. Basically, the Wilcoxon test indicates the differences in the performance of two techniques for each data set and compares the ranks for the positive and negative differences. Such a test is recommended in [38] and has been widely adopted in the literature of machine learning to evaluate and compare between techniques over multiple data sets. In the statistical tests performed in this manuscript, a confidence level of $95 \%(\alpha=0.05)$ has been 
considered.

\subsection{Experiments on artificial data sets}

In this section, we present empirical analysis of the optimization framework on artificial data sets characterized by distinct levels of mixture between the $m=100$, gen $=100$ and $q=3$.

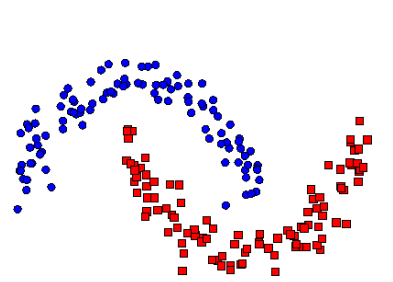

(a) TwoMoons-10

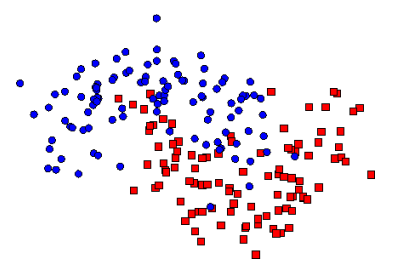

(b) TwoMoons-25

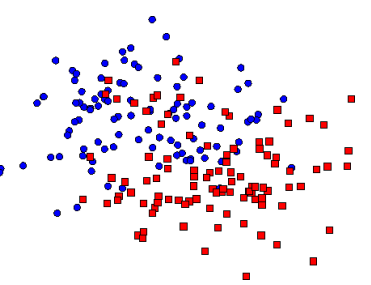

(c) TwoMoons-50

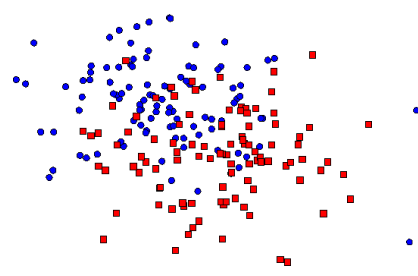

(d) TwoMoons-75

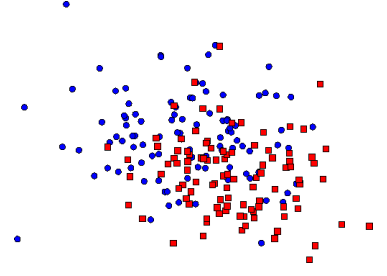

(e) TwoMoons-90

Figure 5: Artificial data sets generated with distinct level of mixture between the classes. (a) $10 \%$; (b) $25 \%$; (c) $50 \%$; (d) $75 \%$; (e) $90 \%$.

Table 1 presents the results obtained by using the two network formation methods and the optimization framework over the five data sets with distinct 
Table 1: Results obtained by the proposed framework over the five data sets, presented in Fig. 5 characterized by distinct level of mixture between the classes. The optimization framework is compared with two network formation methods widely used in literature, $k \mathrm{NN}$ and $k \mathrm{AOG}$.

\begin{tabular}{rlll}
\hline Data set & $k$ AOG & $k$ NN & BIO \\
\hline \hline TwoMoons-10 & $\mathbf{1 0 0 . 0} \pm \mathbf{0 . 0 0}$ & $99.50 \pm 0.15$ & $99.80 \pm 0.06$ \\
TwoMoons-25 & $88.00 \pm 7.14$ & $89.00 \pm 6.24$ & $\mathbf{8 9 . 8 0} \pm \mathbf{6 . 7 9}$ \\
TwoMoons-50 & $74.50 \pm 11.06$ & $\mathbf{8 1 . 5 0} \pm \mathbf{9 . 2 3}$ & $80.70 \pm 7.99$ \\
TwoMoons-75 & $71.50 \pm 6.73$ & $74.50 \pm 8.50$ & $\mathbf{7 7 . 2 0} \pm \mathbf{6 . 4 2}$ \\
TwoMoons-90 & $67.80 \pm 11.00$ & $70.50 \pm 12.54$ & $\mathbf{7 2 . 2 0} \pm \mathbf{1 1 . 0 4}$ \\
\hline
\end{tabular}

\subsection{Experiments on real-world data sets}

This section evaluates our bio-inspired framework over ten real-world data sets. SubSect. 4.3.1 provides a detailed analysis about framework convergence and performance using both mapping heuristics proposed in this work; SubSects. 4.3 .2 and 4.3 .3 analyze the results of the optimization framework in comparison with directed and undirected network formation methods, respectively. The predictive performance of the framework is compared with very well-known classification techniques in SubSect. 4.3.4. Finally, Sub-sect. 4.3.5 shows details about the optimization process as well as an example of misclassification case for traditional techniques. 
The real-world data sets are presented in Table 2 with a brief description. Such a selection was made to encompass diversity on data domains, features and classes. A detailed description about the data sets can be found in 39, 40. As a data preparation, data sets where the predictive attributes are not on similar scale were standardized by taking zero mean and unitary standard deviation, otherwise each instance is normalized by the 12 norm. The euclidean distance was used in all simulations as the distance measurement.

Table 2: Brief description of the real-world data sets in terms of the number of instances (\#Inst.), number of attributes (\#Attr.) and number of classes (\#Classes).

\begin{tabular}{rlll}
\hline Name & \#Inst. & \#Attr. & \#Classes \\
\hline \hline Glass & 214 & 9 & 7 \\
Hayes-roth & 160 & 4 & 3 \\
Iris & 150 & 4 & 3 \\
Leukemia & 100 & 50 & 2 \\
Red-Wine & 1599 & 11 & 6 \\
Seed & 210 & 7 & 3 \\
Sonar & 208 & 60 & 2 \\
Teaching & 151 & 5 & 3 \\
Vehicle & 846 & 18 & 4 \\
Vowel & 990 & 13 & 11 \\
\hline
\end{tabular}

\subsubsection{Mapping heuristics and convergence analysis} ing the two mapping heuristics, MapLab and MapAll, are analyzed in detail.

Tables 3 and 4 show the results obtained by the bio-inspired framework during the optimization (training) phase, using MapLab and MapAll heuristics, respectively. In those tables, the average accuracy and the standard deviation 
tions, respectively. In both tables, we see a considerable improvement in terms of predictive performance in almost all data sets by considering the structural optimization. The Iris data set is an exception as the maximum predictive performance is reached at the first generation. In this case, there is no space for the optimization process.

Table 3: Convergence analysis using MapLab mapping heuristic in the optimization phase. The results represent the average accuracy obtained through the quality function $f$ for one, ten and one hundred generations, respectively.

\begin{tabular}{llll}
\hline Data set & lgen & 10gen & 100gen \\
\hline \hline Glass & $57.67 \pm 3.63$ & $66.15 \pm 2.88$ & $83.11 \pm 1.88$ \\
Hayes-roth & $68.97 \pm 2.76$ & $77.14 \pm 2.81$ & $84.70 \pm 2.29$ \\
Iris & $100.0 \pm 0.00$ & - & - \\
Leukemia & $91.52 \pm 1.82$ & $93.86 \pm 1.47$ & $95.15 \pm 1.37$ \\
Red-Wine & $35.16 \pm 2.43$ & $45.86 \pm 1.54$ & $63.40 \pm 1.26$ \\
Seed & $99.79 \pm 0.63$ & $99.79 \pm 0.63$ & $99.79 \pm 0.63$ \\
Sonar & $74.85 \pm 3.75$ & $79.66 \pm 3.87$ & $85.96 \pm 2.93$ \\
Teaching & $76.52 \pm 4.44$ & $82.56 \pm 3.92$ & $91.44 \pm 4.20$ \\
Vehicle & $70.62 \pm 0.96$ & $73.42 \pm 0.63$ & $83.47 \pm 0.66$ \\
Vowel & $52.24 \pm 3.49$ & $57.39 \pm 3.74$ & $68.77 \pm 5.25$ \\
\hline
\end{tabular}

We also provide detailed results about the convergence process. Fig. 6 shows the average accuracy obtained by the bio-inspired framework during the optimization phase, using MapLab and MapAll heuristics, respectively. In this figure, we see that a population of one hundred particles and one hundred generations are usually sufficient to assure a good convergence for the optimization framework. In addition, MapAll performance is slightly better than MapLab in the optimization phase.

In spite of the results presented in the optimization phase, the predictive accuracy of the structural optimization framework must be evaluated by the 
Table 4: Convergence analysis using MapAll mapping heuristic in the optimization phase. The results represent the average accuracy obtained through the quality function $f$ for one, ten and one hundred generations, respectively.

\begin{tabular}{llll}
\hline Data set & lgen & 10gen & 100gen \\
\hline \hline Glass & $72.12 \pm 3.04$ & $77.45 \pm 3.38$ & $82.22 \pm 2.48$ \\
Hayes-roth & $69.35 \pm 3.47$ & $77.41 \pm 2.76$ & $79.62 \pm 2.91$ \\
Iris & $100.0 \pm 0.00$ & - & - \\
Leukemia & $95.07 \pm 0.81$ & $96.02 \pm 1.04$ & $96.11 \pm 1.04$ \\
Red-Wine & $56.30 \pm 1.25$ & $60.49 \pm 1.14$ & $71.91 \pm 0.83$ \\
Seed & $99.79 \pm 0.63$ & $99.79 \pm 0.63$ & $99.79 \pm 0.63$ \\
Sonar & $75.57 \pm 3.88$ & $81.62 \pm 2.44$ & $88.60 \pm 1.94$ \\
Teaching & $80.05 \pm 4.49$ & $86.89 \pm 5.24$ & $91.05 \pm 5.13$ \\
Vehicle & $72.77 \pm 0.72$ & $76.22 \pm 1.24$ & $85.70 \pm 1.25$ \\
Vowel & $55.86 \pm 2.39$ & $62.74 \pm 2.04$ & $72.34 \pm 3.46$ \\
\hline
\end{tabular}



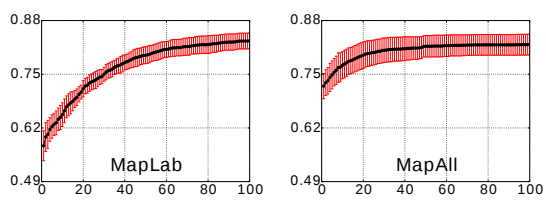

(a) Glass data set
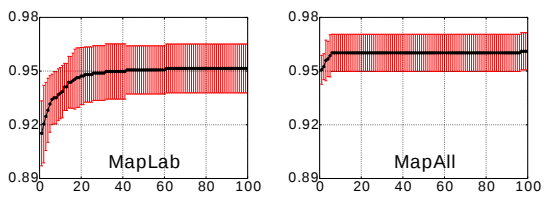

(c) Leukemia data set
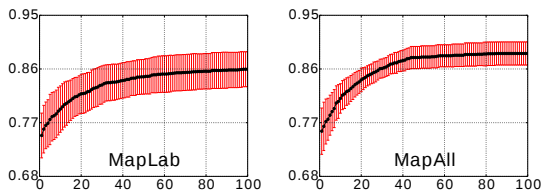

(e) Sonar data set
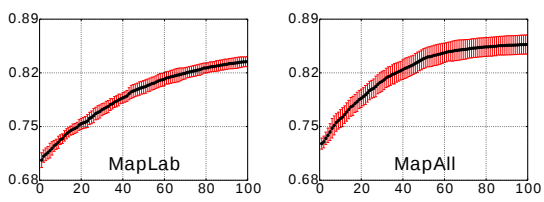

(g) Vehicle data set

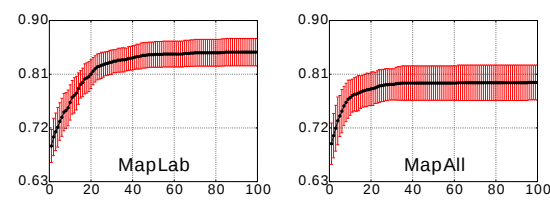

(b) Hayes-roth data set
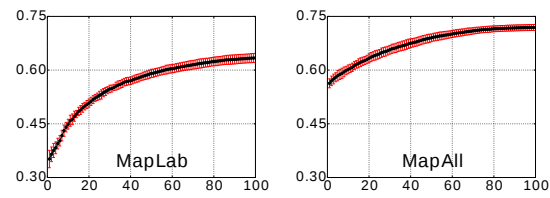

(d) Red-Wine data set
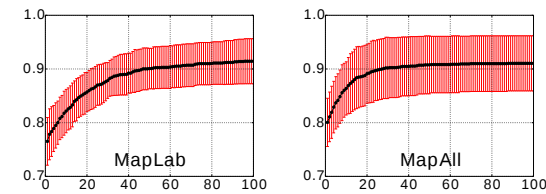

(f) Teaching data set
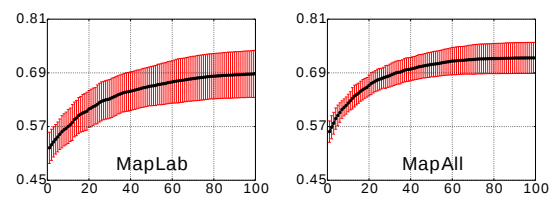

(h) Vowel data set

Figure 6: Analysis of the convergence in the optimization phase of the proposed framework using MapLab and MapAll mapping heuristics. Each subfigure shows the average accuracy obtained through the quality function $f$ as a function of number of generations. The results show that one hundred particles and one hundred generations are usually sufficient to assure the convergence of both heuristics. In terms of predictive performance, MapAll mapping heuristic is slightly better. 

dealing with real-world data sets. In the next computer simulations provided in this manuscript, BIO is equipped with MapAll mapping heuristic.

Table 5: Predictive accuracy obtained by the proposed framework when using MapLab and MapAll mapping heuristics in the test phase.

\begin{tabular}{lll}
\hline Data set & BIO (MapLab) & BIO $($ MapAll $)$ \\
\hline \hline Glass & $60.82 \pm 9.07$ & $\mathbf{6 4 . 5 5} \pm \mathbf{7 . 1 3}$ \\
Hayes-roth & $55.34 \pm 15.17$ & $\mathbf{5 8 . 9 9} \pm \mathbf{1 1 . 8 1}$ \\
Iris & $98.67 \pm 4.00$ & $98.67 \pm 4.00$ \\
Leukemia & $84.22 \pm 8.59$ & $\mathbf{8 6 . 4 7} \pm \mathbf{9 . 6 9}$ \\
Red-Wine & $49.10 \pm 3.24$ & $\mathbf{5 3 . 8 9} \pm \mathbf{3 . 8 6}$ \\
Seed & $\mathbf{9 1 . 0 5} \pm \mathbf{8 . 5 0}$ & $90.86 \pm 7.30$ \\
Sonar & $60.88 \pm 12.68$ & $\mathbf{6 3 . 0 6} \pm \mathbf{1 1 . 8 5}$ \\
Teaching & $\mathbf{5 6 . 1 1} \pm \mathbf{1 6 . 1 3}$ & $55.52 \pm 17.40$ \\
Vehicle & $65.79 \pm 3.22$ & $\mathbf{6 7 . 8 0} \pm \mathbf{3 . 5 2}$ \\
Vowel & $58.36 \pm 6.78$ & $\mathbf{6 2 . 8 7} \pm \mathbf{6 . 9 9}$ \\
\hline
\end{tabular}

\subsubsection{Comparison with directed network formation methods}

The well-known supervised network formation methods $k \mathrm{NN}$ and $k \mathrm{AOG}$ are considered here. $k \mathrm{NN}$ has a unique parameter $k \in\{1,2, \ldots, 30\}$, which is associated with the number of nearest neighbors to consider when connecting a given vertex. $k \mathrm{AOG}$ is a non-parametric network formation method, which builds up the graph by optimizing the purity measure. This category also includes the 
RM method (abbreviation for Randomly Mapped graph), which is a baseline that generates random configurations of networks by using our MapAll mapping heuristic. While BIO optimizes the network iteratively, RM determine the connections randomly, i.e., there is no iterative process. RM has two parameters: the number of random networks $R$ and the maximum number of possible links $q$. We have selected $R=100$ since the swarm size of our framework is ${ }_{475} m=100$, and $q=3$ as the increase of $q$ usually changes the performance considerably because the number of network configurations increases. By contrast, the performance variation of $q$ in $\mathrm{BIO}$ is slighter than RM as the optimization process is able to find good or even optimal network configurations. As RM is a stochastic method, the results of each run are averaged on 5 different runs.

The average classification accuracy obtained by using each network formation method is presented in Tab. 6. From the table, we see that the optimization process conducted by the proposed framework is able to obtain a considerable improvement in terms of predictive performance for some data sets, such as "Sonar" and "Teaching", compared to other network formation methods. The results of the statistical test (Wilcoxon Signed Ranks), presented in the same table, reveal no statistical difference between $k \mathrm{NN}, k \mathrm{AOG}$ and $\mathrm{RM}$, all of which are outperformed by the proposed bio-inspired optimization framework.

\subsubsection{Comparison with undirected network formation methods}

This subsection includes comparison against two widely used network formation methods: $\mathrm{S} k \mathrm{NN}$ and $\mathrm{M} k \mathrm{NN}$. This subsection compares the performance of BIO against two undirected network formation methods, the symmetric $k \mathrm{NN}$ $(\mathrm{S} k \mathrm{NN})$ and the Mutual $k \mathrm{NN}(\mathrm{M} k \mathrm{NN})$ networks. These methods are widely used in unsupervised and semi-supervised learning like data clustering and label propagation. $\mathrm{S} k \mathrm{NN}$ and $\mathrm{M} k \mathrm{NN}$ have the same parameter and range of values as the $k \mathrm{NN}$ network, i.e., $k \in\{1,2, \ldots, 30\}$.

Table 7 presents the predictive performance obtained by each network formation in terms of average classification accuracy. From the table, we can see that the optimized network provided by the framework again is able to obtain 
Table 6: Predictive accuracy of the optimization framework in comparison with widely used directed network formation methods ( $k \mathrm{NN}$ and $k \mathrm{AOG}$ ) and a random method (RM). The last rows presents results of the Wilcoxon Signed Ranks statistical tests: (i) the symbols ">" and " $<$ " indicate a rejection of the null hypothesis, where the algorithm corresponding to its column is respectively better or worse than the algorithm corresponding to its row; (ii) and the symbol " " suggests that both the column algorithm and the row algorithm perform equally well (a failure to reject the null hypothesis at the $95 \%$ confidence level, $\alpha=0.05$ ).

\begin{tabular}{lllll}
\hline Data set & BIO & $k$ NN & $k$ AOG & RM \\
\hline \hline Glass & $64.55 \pm 7.13$ & $61.28 \pm 11.27$ & $64.55 \pm 11.34$ & $63.07 \pm 10.06$ \\
Hayes-roth & $\mathbf{5 8 . 9 9} \pm \mathbf{1 1 . 8 1}$ & $54.24 \pm 13.12$ & $53.54 \pm 13.43$ & $56.79 \pm 11.88$ \\
Iris & $98.67 \pm 4.00$ & $98.67 \pm 4.00$ & $98.67 \pm 4.00$ & $95.33 \pm 5.21$ \\
Leukemia & $86.47 \pm 9.69$ & $83.85 \pm 11.54$ & $\mathbf{8 8 . 0 7} \pm \mathbf{7 . 2 9}$ & $80.05 \pm 8.70$ \\
Red-Wine & $\mathbf{5 3 . 8 9} \pm \mathbf{3 . 8 6}$ & $53.43 \pm 3.93$ & $50.98 \pm 2.44$ & $48.41 \pm 3.07$ \\
Seed & $\mathbf{9 0 . 8 6} \pm \mathbf{7 . 3 0}$ & $90.00 \pm 9.15$ & $85.71 \pm 12.05$ & $90.00 \pm 9.39$ \\
Sonar & $\mathbf{6 3 . 0 6} \pm \mathbf{1 1 . 8 5}$ & $57.33 \pm 15.72$ & $59.16 \pm 14.06$ & $52.29 \pm 12.55$ \\
Teaching & $\mathbf{5 5 . 5 2} \pm \mathbf{1 7 . 4 0}$ & $46.10 \pm 11.07$ & $47.98 \pm 14.16$ & $51.15 \pm 17.89$ \\
Vehicle & $67.80 \pm 3.52$ & $66.20 \pm 3.16$ & $66.42 \pm 3.97$ & $\mathbf{6 9 . 0 0} \pm \mathbf{4 . 5 7}$ \\
Vowel & $\mathbf{6 2 . 8 7} \pm \mathbf{6 . 9 9}$ & $56.87 \pm 11.36$ & $59.70 \pm 10.85$ & $60.40 \pm 9.57$ \\
\hline \hline$k$ NN & $>$ & - & - & - \\
$k$ AOG & $>$ & $\sim$ & - & - \\
RM & $>$ & $\sim$ & $\sim$ & - \\
\hline
\end{tabular}


a considerable improvement in terms of predictive performance for some data sets, such as "Hayes-roth", "Sonar" and "Vowel", compared to the widely used $\mathrm{S} k \mathrm{NN}$ and $\mathrm{M} k \mathrm{NN}$. The statistical test results presented in the same table indicate no statistical difference between $\mathrm{S} k \mathrm{NN}$ and $\mathrm{M} k \mathrm{NN}$, both of which are outperformed by BIO.

Table 7: Predictive accuracy of the optimization framework in comparison with widely used undirected network formation methods ( $\mathrm{S} k \mathrm{NN}$ and $\mathrm{M} k \mathrm{NN}$ ). See label of Table 6 for a complete explanation about the statistical tests.

\begin{tabular}{llll}
\hline Data set & BIO & S $k \mathrm{NN}$ & $\mathrm{M} k \mathrm{NN}$ \\
\hline \hline Glass & $64.55 \pm 7.13$ & $53.76 \pm 14.60$ & $\mathbf{6 4 . 8 5} \pm \mathbf{1 0 . 4 9}$ \\
Hayes-roth & $\mathbf{5 8 . 9 9} \pm \mathbf{1 1 . 8 1}$ & $53.26 \pm 12.71$ & $50.96 \pm 15.55$ \\
Iris & $\mathbf{9 8 . 6 7} \pm \mathbf{4 . 0 0}$ & $98.00 \pm 4.27$ & $96.00 \pm 6.11$ \\
Leukemia & $\mathbf{8 6 . 4 7} \pm \mathbf{9 . 6 9}$ & $81.94 \pm 10.92$ & $86.07 \pm 10.07$ \\
Red-Wine & $\mathbf{5 3 . 8 9} \pm \mathbf{3 . 8 6}$ & $51.82 \pm 5.89$ & $52.80 \pm 3.59$ \\
Seed & $\mathbf{9 0 . 8 6} \pm \mathbf{7 . 3 0}$ & $88.09 \pm 8.04$ & $89.52 \pm 9.94$ \\
Sonar & $\mathbf{6 3 . 0 6} \pm \mathbf{1 1 . 8 5}$ & $50.45 \pm 9.06$ & $57.07 \pm 12.50$ \\
Teaching & $\mathbf{5 5 . 5 2} \pm \mathbf{1 7 . 4 0}$ & $53.37 \pm 10.73$ & $55.32 \pm 12.92$ \\
Vehicle & $67.80 \pm 3.52$ & $67.38 \pm 3.51$ & $\mathbf{6 9 . 0 2} \pm \mathbf{4 . 2 5}$ \\
Vowel & $\mathbf{6 2 . 8 7} \pm \mathbf{6 . 9 9}$ & $55.66 \pm 7.98$ & $55.56 \pm 10.90$ \\
\hline \hline S $k \mathrm{NN}$ & $>$ & - & - \\
M $k \mathrm{NN}$ & $>$ & $\sim$ & - \\
\hline
\end{tabular}

\subsubsection{Comparison with other classification techniques}

This category of experiments includes traditional classification techniques, such as $k$-nearest neighbors, naive Bayes (NB) and Support Vector Machines (SVM). It also includes Convolutional Neural Networks (CNN), which is a stateof-the-art classification techniques. The $k$-NN classifier has a unique parameter $k \in\{1,2, \ldots, 30\}$ related to the number of nearest neighbors to consider in order 
to classify each test item. NB is a non-parametric classification technique, in which the likelihood of the features is assumed to be Gaussian in the experiments here. As $S V M$ has many parameters to be set, the parameter search space is reduced by considering the radial basis function as kernel, and the stopping criterion for the optimization method is defined as the Karush-Kuhn-Tucker violation to be less than $10^{-3}$; the kernel and cost parameters are optimized over the sets $\mu \in\left\{2^{4}, 2^{3}, \ldots, 2^{-10}\right\}$ and $C \in\left\{2^{12}, 2^{11}, \ldots, 2^{-2}\right\}$, respectively. In the case of CNN, we reduce the parameter search space by fixing the network architecture as follows: input layer $\rightarrow$ convolutional layer $1 \rightarrow$ convolutional layer 2 $\rightarrow$ full layer $\rightarrow$ output. The number of filters is and the kernel size are optimized respectively over $f \in\{32,64\}$ and $k s \in\{1,3\}$ in both convolutional layers, and the number of neurons is fixed as 512 in the full layer. We also fixed 500 as the number of epochs (empirically defined in order to avoid overfitting), Adam as the training optimizer, ReLU as the activation function in the convolutional layers and Softmax as the activation function to produce the probability distribution. The learning rate, dropout and batch size are optimized respectively over the sets $\alpha \in\{0.001,0.01,0.1\}, d \in\{0,0.5\}$, and $b \in\{10,50\}$. Other CNN parameters were adopted as the default in the Tensorflow framework [4].

Table 8 presents the average classification accuracy obtained for each classification technique. In order to analyze the results, the Wilcoxon's test is performed to compare each pair of algorithms. The statistical test results, presented in the same table, reveal no statistical difference between $k$-NN, NB and SVM; it shows CNN outperformed $k$-NN and NB and has no statistical difference to SVM and BIO; and it also shows that $k$-NN and NB are outperformed by BIO. Despite BIO is statistically equivalent to CNN and SVM, it has much less parameters to be tuned. In addition, although BIO considers physical features of the data like the other techniques under comparison, it is also able to take into account structural properties of the data. In that sense, these results are very promising because they reveal that our network optimization framework can make topological features even more salient, resulting in competitive performance even against state-of-the-art classification techniques and even on 
Table 8: Predictive accuracy of the optimization framework in comparison with very wellknown classification techniques ( $k$-NN, NB, SVM and CNN). See label of Table 6 for a complete explanation about the statistical tests.

\begin{tabular}{llllll}
\hline Data set & BIO & $k$-NN & NB & SVM & CNN \\
\hline \hline Glass & $\mathbf{6 4 . 5 5} \pm \mathbf{7 . 1 3}$ & $61.86 \pm 9.40$ & $42.53 \pm 12.23$ & $61.59 \pm 13.20$ & $62.96 \pm 10.73$ \\
Hayes-roth & $58.99 \pm 11.81$ & $57.23 \pm 9.93$ & $45.94 \pm 10.57$ & $57.91 \pm 12.67$ & $\mathbf{6 9 . 7 9} \pm \mathbf{6 . 4 4}$ \\
Iris & $\mathbf{9 8 . 6 7} \pm \mathbf{4 . 0 0}$ & $97.33 \pm 4.42$ & $96.66 \pm 4.47$ & $95.33 \pm 6.00$ & $95.33 \pm 6.70$ \\
Leukemia & $86.47 \pm 9.69$ & $87.16 \pm 10.58$ & $\mathbf{8 9 . 0 7} \pm \mathbf{9 . 2 7}$ & $88.98 \pm 10.45$ & $87.83 \pm 6.56$ \\
Red-Wine & $53.89 \pm 3.86$ & $52.69 \pm 6.55$ & $51.30 \pm 5.68$ & $\mathbf{5 7 . 5 6} \pm \mathbf{6 . 4 2}$ & $56.74 \pm 7.51$ \\
Seed & $90.86 \pm 7.30$ & $89.52 \pm 8.19$ & $88.09 \pm 10.91$ & $89.52 \pm 11.02$ & $\mathbf{9 3 . 7 1} \pm \mathbf{7 . 1 3}$ \\
Sonar & $\mathbf{6 3 . 0 6} \pm \mathbf{1 1 . 8 5}$ & $51.14 \pm 14.12$ & $58.59 \pm 21.37$ & $54.94 \pm 10.97$ & $53.38 \pm 1.23$ \\
Teaching & $\mathbf{5 5 . 5 2} \pm \mathbf{1 7 . 4 0}$ & $51.41 \pm 14.51$ & $48.38 \pm 9.74$ & $49.54 \pm 13.39$ & $53.43 \pm 17.33$ \\
Vehicle & $67.80 \pm 3.52$ & $69.72 \pm 5.17$ & $49.20 \pm 4.63$ & $\mathbf{8 2 . 8 6} \pm \mathbf{1 . 5 9}$ & $81.24 \pm 1.95$ \\
Vowel & $62.87 \pm 6.99$ & $52.93 \pm 9.33$ & $56.36 \pm 7.47$ & $\mathbf{7 2 . 1 2} \pm \mathbf{1 1 . 5 5}$ & $69.19 \pm 6.68$ \\
\hline \hline$k$-NN & $>$ & - & - & - & - \\
NB & $>$ & $\sim$ & - & - & - \\
SVM & $\sim$ & $\sim$ & $\sim$ & - \\
CNN & $\sim$ & $<$ & $<$ & - & - \\
\hline
\end{tabular}

data sets strongly determined by physical features.

\subsubsection{Network analysis}

In the following we provide some analysis of the networks obtained by the proposed framework. Fig. 7 shows a comparison between networks formed by $k \mathrm{NN}, \mathrm{RM}$ and the proposed framework. The networks are constructed over the "Teaching" data set considering the first run in the experiments. As the number of features is bigger than three, we have used the Fruchterman-Reingold layout [42] to generate the graphs. The $k \mathrm{NN}$ and RM networks are presented by Figs.

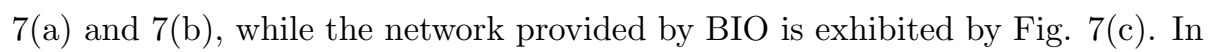
those networks, each data item is represented by a node and each color denotes a class. As already having been pointed out, the optimized network presents the best performance in terms of predictive accuracy. In order to provide a more detailed analysis, we extract information from a component composed by the same nodes in the three networks. In addition, we draw the nodes in 


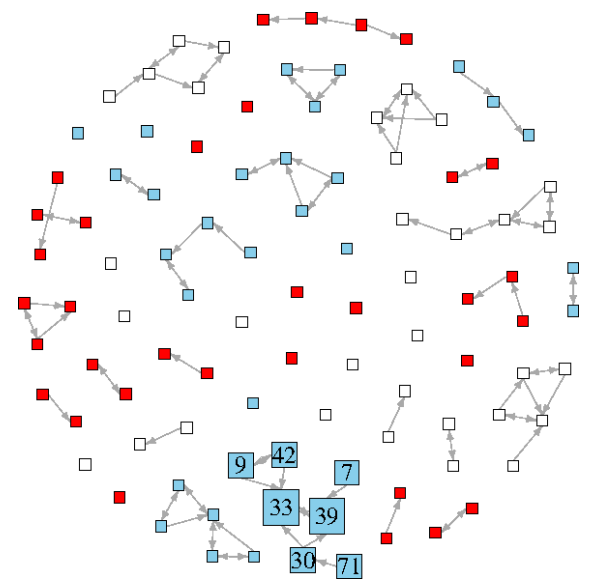

(a) $k \mathrm{NN}(\mathrm{k}=3)$

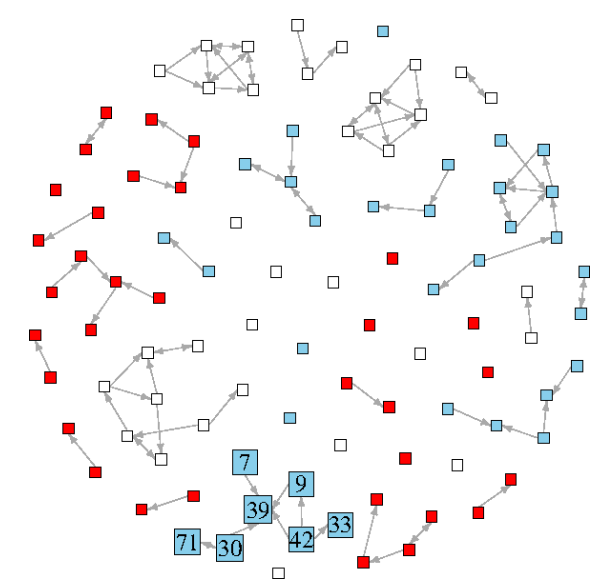

(b) $\mathrm{RM}$

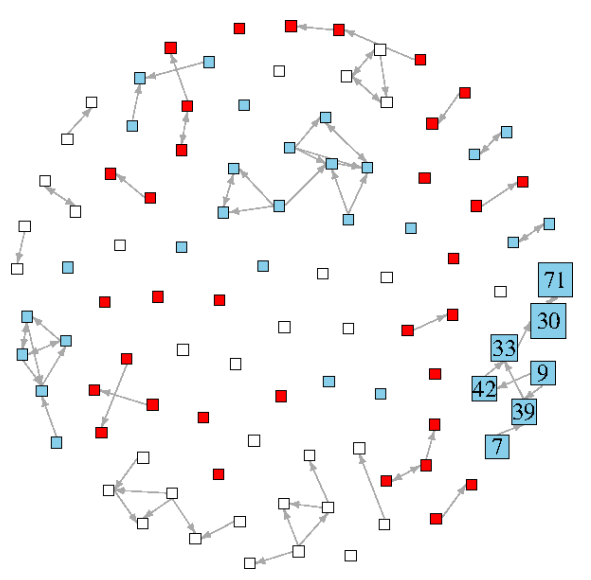

(c) $\mathrm{BIO}$

Figure 7: Comparison between networks formed by different methods. (a) Network obtained by $k \mathrm{NN}$; (b) Network formed by RM; (c) Network provided by BIO. Information extracted from a component composed by the same nodes in the three networks emphasizes a very distinct organizational structure. For example, in $k \mathrm{NN}, \mathrm{RM}$ and BIO networks, the most important nodes in that component have score 0.047 (id. 33), 0.014 (id. 39) and 0.066 (id. $30)$, respectively. 

is provided for each one of them. The most important nodes have scored 0.047 (id. 33), 0.014 (id. 39) and 0.066 (id. 30) in the networks provided by $k \mathrm{NN}$, RM and BIO, respectively. Such an analysis suggests that our framework is promising to find a more efficient organizational structure for the networked data.

Now we move to the other analysis which is illustrated by the optimized network presented by Fig. 8, The figure shows the network obtained for the "Sonar" data set after the optimization process. Circle and square data items represent the two classes of data. The dashed contour data items denote particular test instances wrongly classified by the techniques under comparison. By contrast, the proposed framework is able to assign the correct label for each one of them, since the final classification is given by examining the topological and physical structures of the data according to the optimized network.

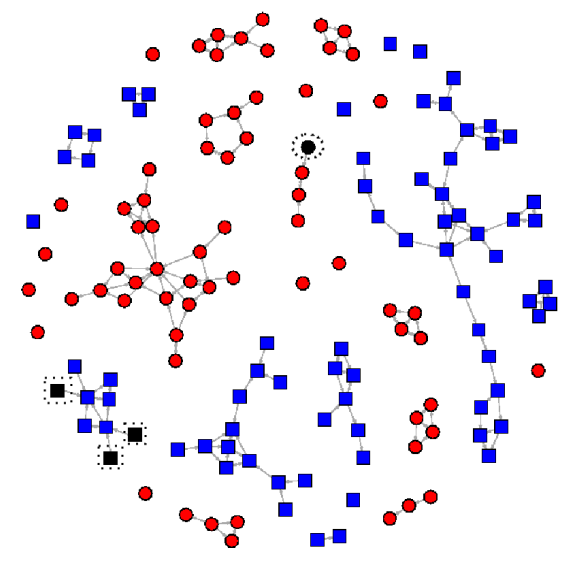

Figure 8: An optimized network obtained for "Sonar" data set. Circle and square data items comprehend the two classes of data. Test instances (dash contoured) denote misleading cases when applying the classification techniques under comparison. 


\section{Discussion and conclusions}

570 original data sets by some optimization process present better performance than those generated heuristically. Generally speaking, the results obtained in this manuscript reveal the advantages of the structural optimization for networkbased classification. In comparison to well-known network formation methods,

\section{Acknowledgment}


2015/50122-0). Authors also acknowledge support from the Brazilian Coordination for the Improvement of Higher Education - CAPES, and the Brazilian National Council for Scientific and Technological Development - CNPq.

\section{References}

[1] S. H. Strogatz, Exploring complex networks, Nature 410 (6825) (2001) 268276.

[2] R. Albert, A.-L. Barabási, Statistical mechanics of complex networks, Reviews of modern physics 74 (1) (2002) 47.

[3] S. N. Dorogovtsev, J. F. Mendes, Evolution of networks, Advances in physics 51 (4) (2002) 1079-1187.

[4] M. E. Newman, The structure and function of complex networks, SIAM review 45 (2) (2003) 167-256.

[5] S. Boccaletti, V. Latora, Y. Moreno, M. Chavez, D.-U. Hwang, Complex networks: Structure and dynamics, Physics reports 424 (4) (2006) 175-308.

[6] D. J. Watts, S. H. Strogatz, Collective dynamics of small-worldnetworks, nature 393 (6684) (1998) 440-442.

[7] A.-L. Barabási, R. Albert, H. Jeong, Scale-free characteristics of random networks: the topology of the world-wide web, Physica A: Statistical Mechanics and its Applications 281 (1) (2000) 69-77.

[8] R. Pastor-Satorras, A. Vespignani, Epidemic spreading in scale-free networks, Physical review letters 86 (14) (2001) 3200.

[9] M. Newman, Networks: An Introduction, Oxford University Press, Inc., 2010.

[10] R. F. i Cancho, R. V. Solé, Optimization in complex networks, in: Statistical mechanics of complex networks, Springer, 2003, pp. 114-126. 
[11] T. C. Silva, L. Zhao, Machine learning in complex networks, Springer, 2016.

[12] K. Ozaki, M. Shimbo, M. Komachi, Y. Matsumoto, Using the mutual knearest neighbor graphs for semi-supervised classification of natural language data, in: Proceedings of the fifteenth conference on computational natural language learning, Association for Computational Linguistics, 2011, pp. $154-162$.

[13] T. C. Silva, L. Zhao, Network-based high level data classification, IEEE Transactions on Neural Networks 23 (2012) 954-970.

[14] T. Jebara, J. Wang, S.-F. Chang, Graph construction and b-matching for semi-supervised learning, in: Proceedings of the 26th Annual International Conference on Machine Learning, ACM, 2009, pp. 441-448.

[15] F. Wang, C. Zhang, Label propagation through linear neighborhoods, IEEE Transactions on Knowledge and Data Engineering 20 (1) (2008) 55-67.

[16] T. H. Cupertino, J. Huertas, L. Zhao, Data clustering using controlled consensus in complex networks, Neurocomputing 118 (2013) 132-140.

[17] B. Araujo, L. Zhao, Data heterogeneity consideration in semi-supervised learning, Expert Systems with Applications 45 (2016) 234-247.

[18] J. R. Bertini, L. Zhao, R. Motta, A. A. Lopes, A nonparametric classification method based on k-associated graphs, Information Sciences 181 (24) (2011) 5435-5456.

[19] G. Palla, I. Derényi, I. Farkas, T. Vicsek, Uncovering the overlapping community structure of complex networks in nature and society, Nature 435 (7043) (2005) 814-818.

${ }_{645}$ [20] O. Chapelle, B. Schölkopf, A. Zien, et al., Semi-supervised learning, Vol. 2, MIT press Cambridge, MA:, 2006.

[21] S. Yan, D. Xu, B. Zhang, H.-J. Zhang, Q. Yang, S. Lin, Graph embedding and extensions: A general framework for dimensionality reduction, IEEE 
Transactions on Pattern Analysis and Machine Intelligence 29 (1) (2007) $40-51$.

[22] X. Zhu, Semi-supervised learning literature survey, Tech. rep., University of WisconsinMadison (2008).

[23] S. Fortunato, Community detection in graphs, Physics Reports 486 (3-5) (2010) $75-174$.

[24] A. Goldenberg, A. X. Zheng, S. E. Fienberg, E. M. Airoldi, A survey of statistical network models, Foundations and Trends@ in Machine Learning 2 (2) (2010) 129-233.

[25] T. C. Silva, L. Zhao, Network-based stochastic semisupervised learning, IEEE Transactions on Neural Networks and Learning Systems 23 (3) (2012) 451-466.

[26] M. E. Newman, Communities, modules and large-scale structure in networks, Nature Physics 8 (1) (2012) 25-31.

[27] T. C. Silva, L. Zhao, Uncovering overlapping cluster structures via stochastic competitive learning, Information Sciences 247 (2013) 40-61.

[28] M. G. Carneiro, T. H. Cupertino, L. Zhao, K-associated optimal network for graph embedding dimensionality reduction, in: IEEE International Joint Conference on Neural Networks (IJCNN), 2014, pp. 1660-1666.

[29] M. G. Carneiro, J. L. Rosa, A. A. Lopes, L. Zhao, Network-based data classification: combining k-associated optimal graphs and high-level prediction, Journal of the Brazilian Computer Society 20 (1) (2014) 14.

[30] T. H. Cupertino, L. Zhao, M. G. Carneiro, Network-based supervised data classification by using an heuristic of ease of access, Neurocomputing 149 (2015) 86-92. 
[31] T. H. Cupertino, M. G. Carneiro, Q. Zheng, J. Zhang, L. Zhao, A scheme for high level data classification using random walk and network measures, Expert Systems with Applications 92 (2017) 289-303.

[32] M. G. Carneiro, L. Zhao, Organizational data classification based on the importance concept of complex networks, IEEE Transactions on Neural Networks and Learning Systems PP (99) (2017) 1-13.

[33] R. Cheng, Y. Jin, A social learning particle swarm optimization algorithm for scalable optimization, Information Sciences 291 (2015) 43-60.

[34] S. Brin, L. Page, The anatomy of a large-scale hypertextual web search engine, Computer networks and ISDN systems 30 (1) (1998) 107-117.

[35] J. Kennedy, R. Eberhart, Particle swarm optimization, in: Proceedings of the IEEE International Conference on Neural Networks, Vol. 4, IEEE, 1995, pp. 1942-1948.

[36] J. Kennedy, Swarm Intelligence, Springer, 2006.

[37] J. Chen, H.-r. Fang, Y. Saad, Fast approximate k nn graph construction for high dimensional data via recursive lanczos bisection, The Journal of Machine Learning Research 10 (2009) 1989-2012.

[38] J. Demšar, Statistical comparisons of classifiers over multiple data sets, Journal of Machine Learning Research 7 (2006) 1-30.

[39] C. Haslinger, N. Schweifer, S. Stilgenbauer, H. Döhner, P. Lichter, N. Kraut, C. Stratowa, R. Abseher, Microarray gene expression profiling of b-cell chronic lymphocytic leukemia subgroups defined by genomic aberrations and vh mutation status, Journal of Clinical Oncology 22 (19) (2004) 3937-3949.

[40] A. Frank, A. Asuncion, UCI machine learning repository (2010). URL http://archive.ics.uci.edu/ml 
[41] M. Abadi, P. Barham, J. Chen, Z. Chen, A. Davis, J. Dean, M. Devin, S. Ghemawat, G. Irving, M. Isard, et al., Tensorflow: a system for largescale machine learning., in: USENIX Symposium on Operating Systems Design and Implementation, Vol. 16, 2016, pp. 265-283.

[42] T. M. Fruchterman, E. M. Reingold, Graph drawing by force-directed placement, Software: Practice and experience 21 (11) (1991) 1129-1164. 